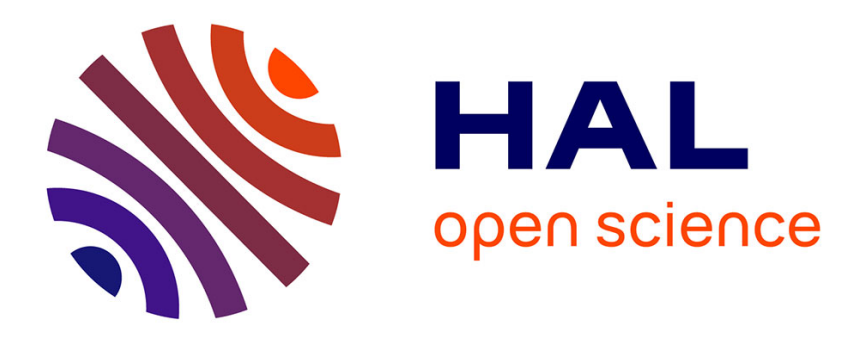

\title{
Consistent 3D geometrical model of fabric elementary cell. Application to a meshing preprocessor for 3D finite element analysis
}

Gilles Hivet, Philippe Boisse

\section{- To cite this version:}

Gilles Hivet, Philippe Boisse. Consistent 3D geometrical model of fabric elementary cell. Application to a meshing preprocessor for 3D finite element analysis. Finite Elements in Analysis and Design, 2005, 42 (1), pp.25-49. 10.1016/j.finel.2005.05.001 . hal-00020539

\section{HAL Id: hal-00020539 \\ https://hal.science/hal-00020539}

Submitted on 17 Apr 2018

HAL is a multi-disciplinary open access archive for the deposit and dissemination of scientific research documents, whether they are published or not. The documents may come from teaching and research institutions in France or abroad, or from public or private research centers.
L'archive ouverte pluridisciplinaire HAL, est destinée au dépôt et à la diffusion de documents scientifiques de niveau recherche, publiés ou non, émanant des établissements d'enseignement et de recherche français ou étrangers, des laboratoires publics ou privés. 


\title{
Consistent 3D geometrical model of fabric elementary cell. Application to a meshing preprocessor for 3D finite element analysis
}

\author{
Gilles Hivet $^{\mathrm{a}, *}$, Philippe Boisse ${ }^{\mathrm{a}, \mathrm{b}}$ \\ ${ }^{a}$ Laboratoire de Mécanique de Systèmes et des Procédés, UMR CNRS 8106, ENSAM-ESEM, \\ 8 rue Léonard de Vinci 45072 Orléans, Cedex, France \\ ${ }^{\mathrm{b}}$ Laboratoire de Mécanique des Contacts et des Solides UMR CNRS 5514, INSA de Lyon Bâtiment Jacquard, \\ Rue Jean Capelle, 69621 Villeurbanne, Cedex, France
}

Many different methods can be used to obtain a composite part. Among them, several require the forming of a dry fabric before the resin is injected. The mechanical properties of the obtained part depend to a large extent on the mechanical behavior of the dry fabric. Experimental methods are efficient to identify this behavior, but they need to be complemented with numerical approaches. Among the numerical approaches, 3D finite element simulation is very interesting but requires an accurate 3D mesh of the fabric elementary cell. In this paper, we are proposing a new consistent 3D geometrical model of 2D fabrics. Experimental observations using different optical processes have been performed in order to determine real yarn geometry in different cases of yarn structure and weaving. The analysis of these results helps us define the accurate generic 3D model of a yarn shape when it is weaved. Using this model of a yarn, a consistent 3D geometrical model of 2D fabrics is presented. One particularity of this model is that it ensures a realistic contact surface between yarns without interpenetration for all types of weaving. The section shape varies along the trajectory, so that the influence of contact between yarns on their cross section shape can be taken into account. This model can be easily identified using three to seven parameters measured on a real fabric. A meshing preprocessor based on this geometrical model is then developed. It substantially reduces the time needed to obtain an accurate hexahedral mesh of the elementary cell of a fabric. This is an important point for the 3D finite element

\footnotetext{
* Corresponding author. Tel.: +3323841 70 56; fax: +3323841 7063 .

E-mail address: gilles.hivet@univ-orleans.fr (G. Hivet).
} 
simulation of fabrics, which is a powerful method to investigate their mechanical behavior. Examples of shear and biaxial extension are presented to show the efficiency of the whole simulation chain.

Keywords: Finite elements; Fabric; Geometrical mesoscopical model; Preprocessor; Mesh

\section{Introduction}

The finite element analysis of the unit cell of a fabric is a difficult problem encountered in different types of study. A direct analysis of the mechanical response of the fabric cell to loadings (or deformations) allows to obtain its mechanical behavior without proceeding to experiments that are often difficult. The loading can be biaxial extension [1,2], or plane shear [3-5], the mechanical characteristics are then used in simulations of tensed woven reinforced fabric structures, or in forming simulations [4,6-9]. Another indirect type of analysis consists in identifying voids between yarns to analyze resin flow during an injection phase [10-12]. Permeability of the reinforcement is then deduced from this local flow study and can be used in an injection simulation of an entire part $[13,14]$. The simulation of a unit cell deformation needs an accurate mesh of the unit cell. Defining the geometry on which this mesh is applied is a difficult and important point. The multi-scale nature of the fabric (macro-scale), composed of yarns (meso-scale), themselves composed of fibers (micro-scale) leads to a complicated geometry that is difficult to identify. A simplified model should be used for the meshing geometrical support. Numerous models exist [15-18]. Nevertheless, meshes obtained with these models are generally not usable to perform a finite element analysis of the unit cell. Those models take into account contact between yarns, so, contact surfaces must be very precisely defined. Interpenetration between yarns is redhibitory for such an analysis. Likewise, the existence of unreal voids (due to the modelling) significantly affects finite element results. The goal of this study is to present a tool for the definition of a consistent 3D geometrical model of fabric reinforced unit cells. The model is said to be consistent because it guarantees there is no penetration between warp and weft yarns, and it imposes that contact happens where it should take place. Assumptions for this model are based on 2D and 3D experimental observations of fabric reinforcements used for composite materials. All standard weaving types (plain weave, twill, satin) are studied. This model is then used to define the unit cell mesh. This mesh is then used to perform finite element simulations of the unit cell mechanical behavior. Models for different weavings are presented, as well as a biaxial tension analysis on a glass plain weave.

\section{Description of yarn structure and mechanical behavior}

\subsection{Yarn structure and geometry}

Yarns are composed of an assembly of fibers. There are many types of fibers: their constitutive material (carbon, glass, etc.), geometry (diameter, length), for example, confer them radically different mechanical properties. Different processes can be used to obtain a yarn out of fibers [19]. The fibers can be juxtaposed, twisted, or entangled. They can also be coated. The volume fraction of fibers in a yarn (ratio of the fibers volume to the yarn volume), but also their geometry and mechanical properties are very variable [19]. The 


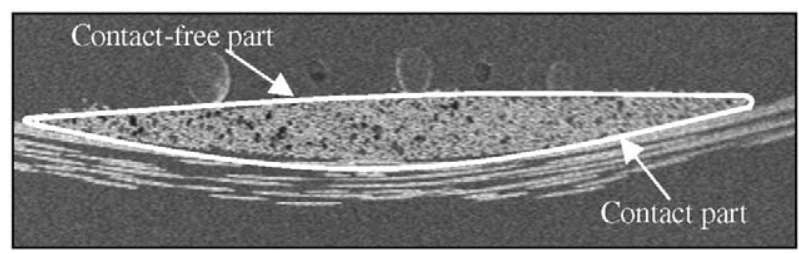

Fig. 1. Definition of a yarn section.

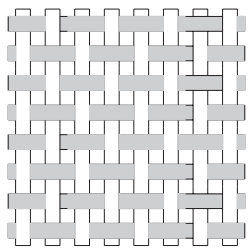

(a) Plain weave

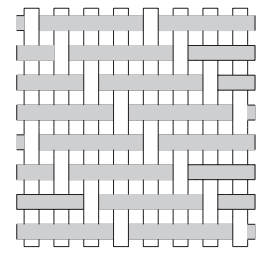

(b) Twill $3 * 1$

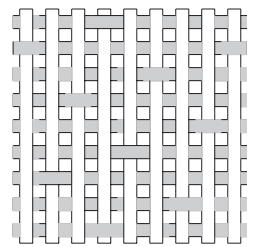

(c) Satin 8

Fig. 2. Different types of standard weaving: (a) plain weave, (b) twill $3 * 1$ and (c) satin 8 .

cohesion of fibers inside the yarn is very dependent on the manufacturing process. The reorganization of fibers is a key point to understand the evolution of a yarn's geometry. The study of fabric mechanical behavior needs to take this great diversity into account. If the section of a yarn is defined as the envelope of the fibers (Fig. 1), yarn sections strongly depend on the yarn structure. Some yarns have an intrinsic section that is quite constant with low solicitations, whereas for some others, the section mostly depends on the boundary conditions.

A powerful geometrical model should be able to represent all types of sections and section evolution. A fabric is obtained by the interlacing of yarns. Many schemes exist. Yarns may be oriented in one, two or more directions. We will only focus on fabrics with yarns oriented in two directions-which are sometimes called 2D fabrics - because they are mostly used for the stamping of complex shapes. Inside this $2 \mathrm{D}$ fabric category, five interlacements are commonly constructed:

- fiber matts;

- woven fabrics: plain, twill and satin weave (Fig. 2);

- knitted fabrics.

Fiber matts are almost isotropic, and knitted fabrics are off the subject of this study [20]. It is important to note that the structure of a plain weave is identical to that of a twill $1 * 1$, and the structure of a satin $n$ is very close to that of a twill $1 * n$. Therefore, developments presented hereafter will mainly be based on twill $m * n$ fabric materials. The weaving of fabrics, that is, values of $m$ and $n$ for a twill $m * n$, mainly influences the yarns' geometry and fibers organization at the initial state.

\subsection{Mechanical properties of fabrics}

Fabrics have a particularity called anisotropy: the rigidity of the material they make up is much higher in the yarn direction than in any other. In usual 2D fabrics, yarns are orientated in two directions only, warp 


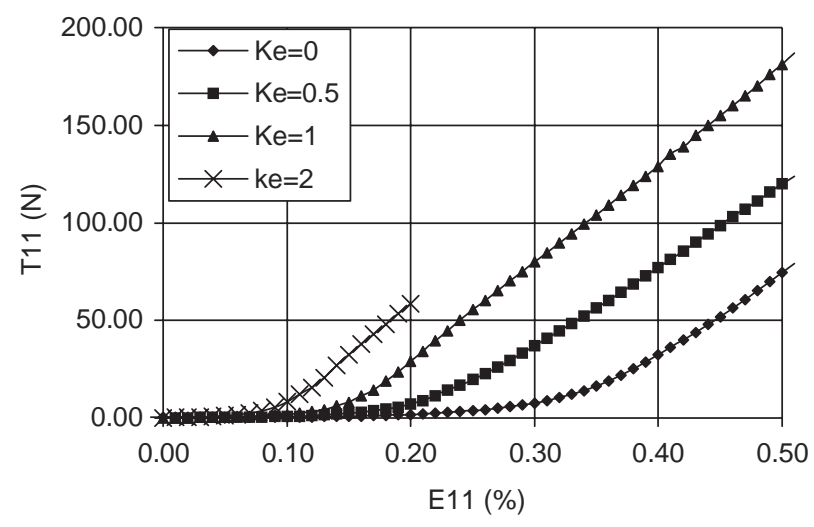

Fig. 3. Experimental bi-axial curves for a balanced carbon twill weave. Tension in weft direction (T11) as a function of strain in weft direction (E11), Ke is the ratio between strains (E22) and (E11).

and weft. The fabric is made up of the interlacement of warp and weft yarns. Identifying the mechanical behavior of such a fabric consists in establishing the relationship between the two tensions and the two deformations in the yarn directions. Experimental biaxial tensile tests on a cross shape specimen have been performed in LMSP in order to obtain this relationship [21,22]. Some results obtained from these experiments are presented hereafter (Fig. 3), for a balanced carbon twill $2 * 2$ weave.

For all tested fabrics, experiments have shown that tension in one direction depends on deformations in the two directions, which is typical of a biaxial behavior. Experiments have also highlighted that even though the fiber tensile law is almost linear for some fabrics, the biaxial tensile laws are not (Fig. 3). The biaxial tensile behavior can be represented as 2 surfaces defining each of the two tensions as a function of the two deformations. Identifying the behavior of a fabric consists in determining what will be called the two tension surfaces. For a balanced fabric, only one is necessary. Different methods can be carried out to get these surfaces. Among them, the experimental one is the most reliable. Various fabrics have been tested, and test results stored in an experimental database [21]. An example of surface obtained with the repartition of experimental points in the deformation space is presented in Fig. 4. Some limitations pertaining to the use of the experimental method must nevertheless be considered. In order to accurately identify the surfaces, many experimental points are needed. Therefore, if a high accuracy is needed for the surface interpolation of experimental points, these should be well distributed, so that control points are available everywhere in the strain domain. Unfortunately, as shown in Fig. 4, experiments do not give equally distributed points on the strain domain. It is especially difficult to obtain points on the surface boundaries, i.e. points with a very low or very high $\varepsilon_{11} / \varepsilon_{22}$ ratio.

Moreover, the experimental approach does not allow the study of fabrics that do not exist. This is an important limitation to the use of this approach, whereas simulation may be used both to optimize existing fabrics or create new fabrics.

\section{Mesoscopic geometrical model of fabrics}

Although the experimental method is the most natural approach, it has some drawbacks. For that reason, we are proposing to complement it with a numerical approach based on the mesoscopic mechanical modeling of fabrics. There are two types of mesoscopic models. For the first type, yarns are assumed to 


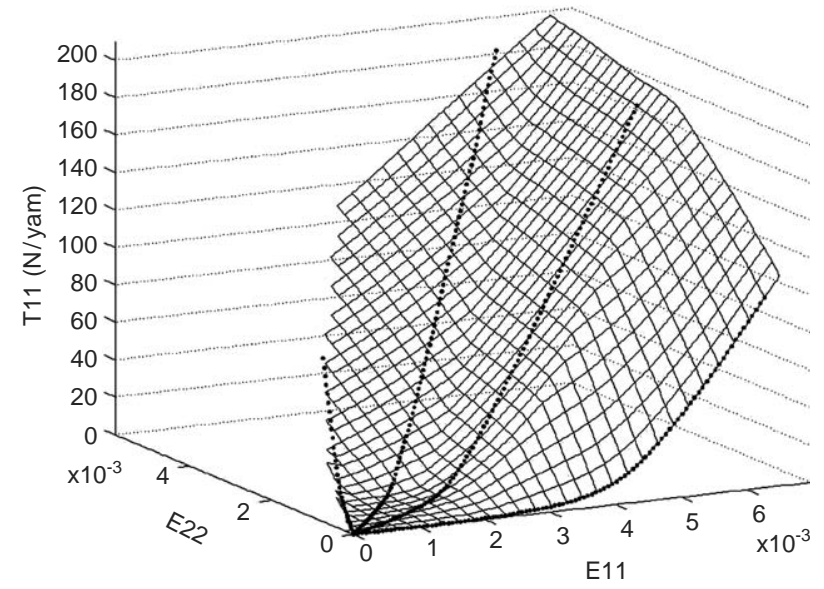

Fig. 4. Experimental behavior surface (partial) for a balanced carbon twill weave. Position of the experimental points on the surface.

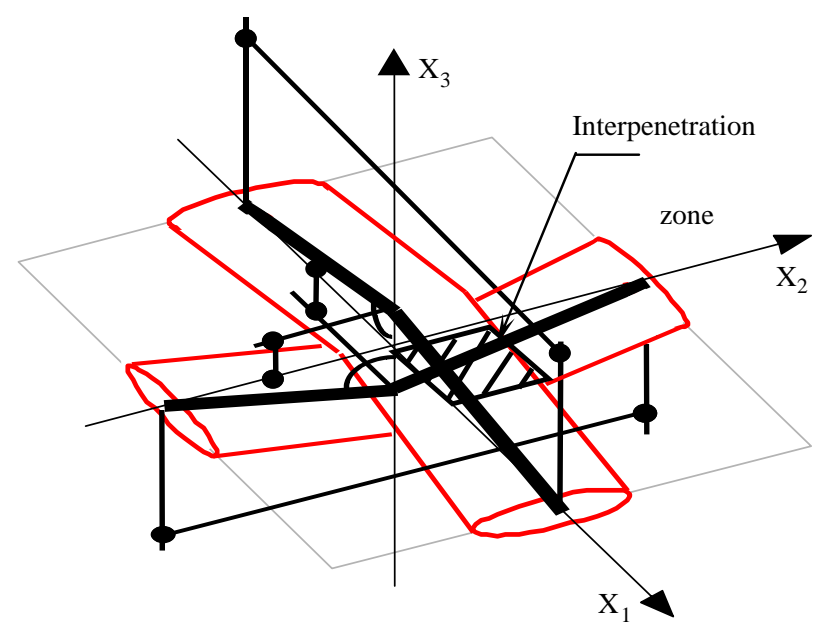

Fig. 5. Articulated straight segment model (Kawabata).

be a composition of hinged rods. These rods can be deformable [15,23], or rigidities can be introduced by springs [24]. The Kawabatta model is well known: it is simple and efficient, but it is not 3D consistent.

If the 3D model of each yarn is constructed through the weep of a section along the trajectory composed of the Kawabatta 2D bars (Fig. 5), Kawabatta 3D model consists in four straight bars (Fig. 5) orientated in four different directions. The assembly of the warp and weft yarn leads whether to interpenetration (Fig. 5) or to four contact points far from the centre of the elementary cell. It is clear that this model has not been designed to propose a 3D mesoscopical model of fabrics.

Therefore, coefficients identified for the transverse crushing behavior in order to obtain a good correlation between model and experiment for tension surfaces are not physically consistent [21]. In the second type of model, the fabric is assumed to be a composition of 3D yarns that are supposed to be homogenous. 
Some models of this type are already available [25-27]. Their purpose is to give a better description of the fabric geometry at the initial state. Some of them are based on the principle of a constant section with a curvilinear trajectory. Curves used to represent elements can be sinusoids [16,25-28], splines, circles, or polynomials with elliptic sections. Some results of bi-axial simulation behavior can be found in literature $[27,29]$. The consistency of results coming from different models is presented in [29]. Nevertheless, many of them are not perfectly consistent with others. Some interpenetration can be noticed at the contact zone between warp and weft yarns. Moreover, our experimental observations will show that the assumption there is a constant section along the yarn seems not to be realistic enough. In the model developed by Kuhn and Charalambides, the section varies along the trajectory, taking into account the reorganization of fibers near the contact zone [26]. Consistency seems to be well ensured, but this model is limited to plain weaves and has only been used to generate elementary cells of composite materials. The conclusion of this preliminary study is that the existing models seem to be too geometrically constrained to be completely consistent with experimental observations for all types of weaving. We will propose our own geometrical model for fabrics, which will model the contact zone between yarns much more accurately, in order to ensure a consistent contact surface. We will show its consistency with experimental observations made in our lab or found in literature. We will then show it can be applied to a meshing preprocessor. Finally, we will apply the model to perform 3D finite element simulations on the elementary cell, in order to identify tension surfaces and shear curves.

\section{Experimental observations}

The aim of this first step is to build a geometrical model that can take into account the diversity of fabric geometries. A yarn is assumed to be a continuous solid, but both the geometry and the mechanical behavior of this continuum will take into account the fibrous nature of yarns. A yarn geometrical model is defined by the volume generated by the sweep of a section along a trajectory. The section is always defined by the envelope of yarn cross section (Fig. 1). Trajectory is defined by the longitudinal section of the yarn (Fig. 6a).

\subsection{Experimental observations on yarn section}

The 3D model of the fabric results from the consistent assembly (no interpenetration and no spurious voids) of its yarns. In order to identify the yarn cross section and trajectory, we need to observe the 3D structure of different fabrics. Many methods can be used. 3D measures with contact could be considered, but, as we already mentioned before, for many fabrics, when contact occurs, the cohesion between fibers is not sufficient to ensure the conservation of the cross section shape. Two other options can as well be explored to observe the cross section. It is possible to use optical methods, but only the visible parts of the yarn section can be observed. The yarn needs to be cut so the cross section can be seen. For most fabrics, cutting the yarn leads to a modification of the section (yarns ruffle). We have to coat the dry fabric with some transparent resin to keep the original shape, and only then cut the sample. But here again, the section shape may be modified by the introduction of the resin. It can be seen on micrographs that resin penetrates inside the yarns and between the fibers. This phenomenon is well known among people who study injection problems. As a conclusion, none of these techniques is perfect. So we will confront the 


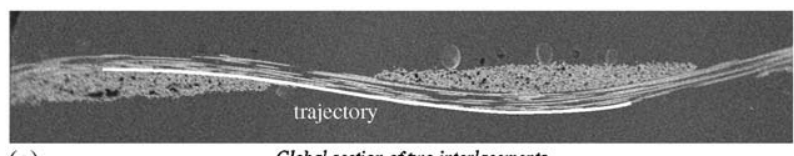

(a)

Global section of two interlacements

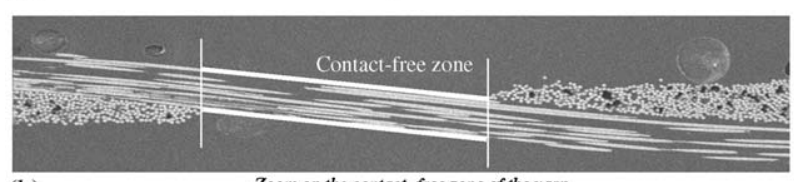

(b)

Zoom on the contact-free zone of the yarn

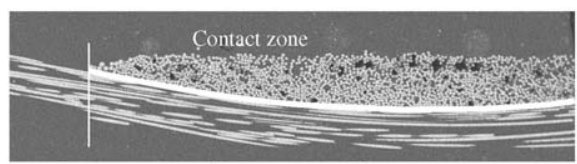

(c)

Zoom on a yarn section

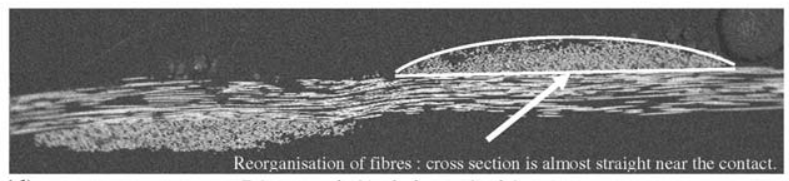

(d)

Fabric stretched in the longitudinal direction

Fig. 6. Transverse cut of a sample of a balanced glass plain weave: (a) global section of two interlacements; (b) zoom on the contact-free zone of the yarn; (c) zoom on a yarn section; and (d) fabric stretched in the longitudinal direction.

results from the two methods that are supposed best: cutting a coated sample and observing the visible parts of the sample with optical methods.

Coated samples have been made in collaboration with Lowell University. Many pictures of two plain weave fabrics - a twintex and a balanced glass plain weave-have been taken with an electronic microscope. Some of them are presented Figs. 6 and 7. Samples have been made with and without pretension, in order for us to see whether the cross section and trajectory are modified.

Three planes are defined: the horizontal plane that is parallel to the fabric plane, the cross section plane, and what will be called the vertical plane, which is orthogonal to the fabric plane and orthogonal to the cross section plane. They are presented in Fig. $7 \mathrm{~b}$.

Observing micrographs of the coated samples leads to the following conclusions: The yarn section is close to a lenticular shape. It is composed of two parts linked through two points. The first part is the contact zone. The second part is the contact-free zone (Fig. 1). It can also be noted that cross sections are dissymmetric according to the horizontal plane (Figs. 6 and 7). This dissymmetry can also be observed on pictures taken on a belt [32]. Both the cross and the longitudinal sections change when a tension is applied in one direction of the fabric (Fig. 6d). The tensed yarn is almost straight whereas the undulation of the free transverse yarn rises. The free yarn section becomes almost flat near the contact. The reorganization of fibers is clearly seen here (Fig. 6d). The yarn section dissymmetry is more important in this case. The evolution of section in function of the tension has also been observed in other papers [23,28,30,31]. As a conclusion, the reorganization of the fibers in the yarn appears to be a very important phenomenon: the cross section is dissymmetric due to the contact, and it changes depending on boundary conditions on the fabric. The second series of tests have been performed with optical measures. Some of the results are presented in Fig. 8 for a carbon twill and a glass plain weave. The particularity of these two fabrics 


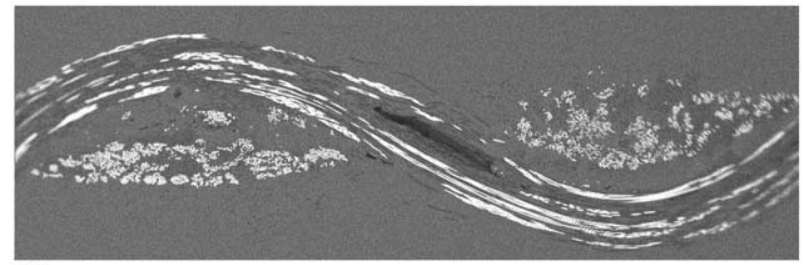

(a)

Global view of two interlacements

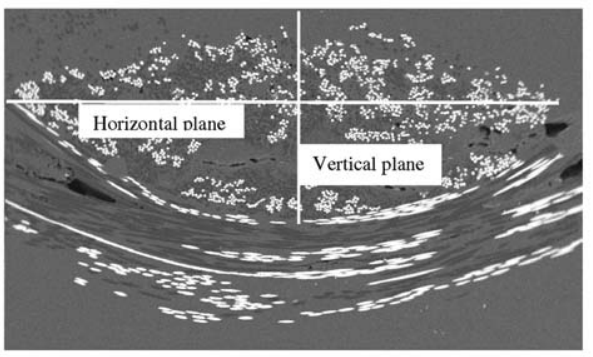

(b)

Zoom on a yarn section in the cross section plane

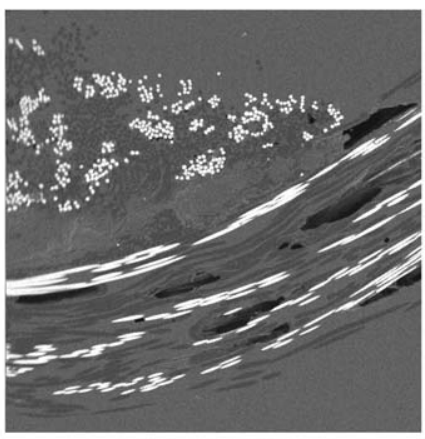

(c)

Zoom on the side of a yarn

Fig. 7. Transverse cut of a twintex sample: (a) global view of two interlacements; (b) zoom on a yarn section in the cross section plane; and (c) zoom on the side of a yarn.

is that the carbon yarns are coated and the glass yarns are twisted. The consequence is higher cohesion between the fibers in the yarn. It can be noted that the cross section is, here again, dissymmetric. The contact zone between the yarns is large and the contact mainly influences the cross section shape. But the reorganization of fibers is not sufficient to get to the lenticular shape previously observed. There are lateral zones between the contact and contact-free sides. The original shape is more stable. This is due to the yarn manufacturing (coating and twisting), which grants higher cohesion to fibers. For plain weaves, the cross section is symmetrical according to the vertical plane (which make sense given the fabric structure).

The conclusion of all these experiments is that three different zones can be differentiated for the cross section: a contact zone, a contact-free zone, and a lateral zone that can be limited to only two points in case of a weak cohesion of fibers (Fig. 9). In the general case, these three zones can be approached through four conic curves for instance (parabolas, circles). Values chosen for the lateral conics parameters will make these vary from straightline to dot. Thus, all types of yarns observed can be represented using this model. For plain weave fabrics, the symmetry according to the vertical plane leads to a simplified form 


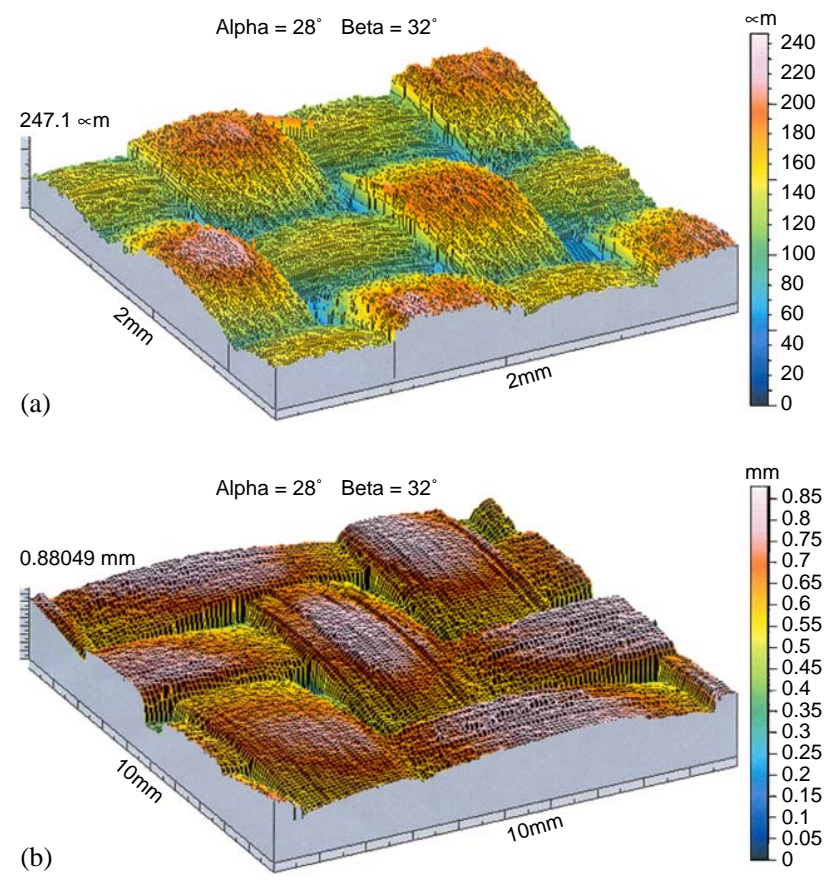

Fig. 8. 3D external optical measure of dry fabrics at the initial state (no tensions): (a) 3D optical measure on an unbalanced glass plain weave and (b) 3D optical measure on a carbon twill $2 * 2$.

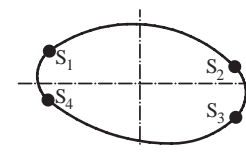

General form

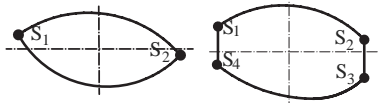

(a)
Simplified forms
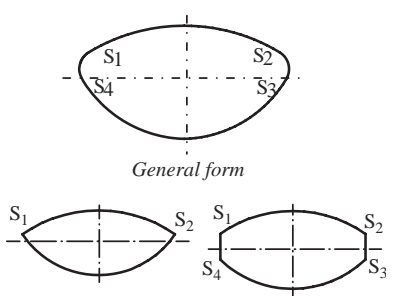

(b)
Simplified forms

Fig. 9. Models for the cross section of a 2D fabric: (a) generic model and (b) plain weave model.

of the cross section. The generic models of sections for twill and plain weave, and the two associated simplified forms are presented in Fig. 9.

\subsection{Experimental observations on yarn "trajectory"}

The trajectory is constrained by the necessary 3D consistency of the fabric model. For plain weaves, the contact zone will consist in the same conic as that of the cross section (Fig. 6b). In the contact-free zone, no lateral load is applied to the yarn. Given that the bending rigidity of yarns is very weak, the contact-free part of the trajectory should be straight (Fig. 6c). So, the trajectory is composed of conics and straight segments. Tangency conditions between conics and segments must be ensured. The geometrical 


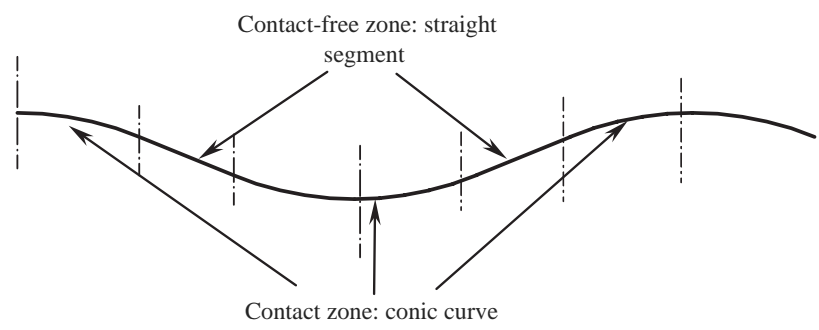

Fig. 10. Model of a trajectory for a plain weave.

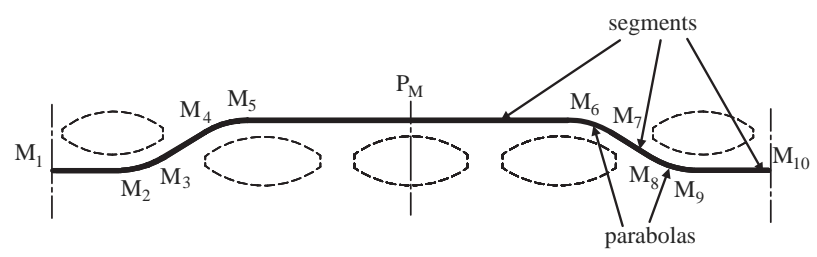

Fig. 11. Model of a trajectory for twill $3 * 2$.

model is presented in Fig. 10. This model matches observations of longitudinal sections in the different pictures presented before (Figs. 6 and 7).

For twills, the problem is a little bit more complex. A yarn passes over $m$ and under $n$ transverse yarns. When this yarn passes over the $m$ transverse yarns, two cases may be considered:

- The yarn follows the original curvature of the transverse yarn cross sections. In this case, the yarn is curved when contact occurs.

- The transverse cross section flattens and the yarn keeps almost straight.

The second assumption is much more consistent with our previous observations (Fig. 8). Moreover, when the fabric is deformed, tension in the fibers will make this second model more suitable. The trajectory obtained for any twill (or satin) is then very simple, which is a good point for model identification. This is presented in Fig. 11.

\section{Yarn 3D model}

Most existing models assume that the cross section of the yarn is constant along the trajectory. The cross section should then be symmetric according to the fabric plane. We have experimentally shown that this is not true. The reorganization of fibers appears to be a significant factor influencing the yarn geometry. The cross section changes depending on the contact location. In the present model, we will take into account the variations of the section shape along the yarn using control sections at control points. The complete 3D model of the yarn is obtained through a smooth interpolation between the control sections, which follows the imposed trajectory. The interpolation is obtained using CAD software, such as PROEngineer ${ }^{\circ}$, which includes a "swept blend" feature that is able to build volumes using control sections and trajectories. In 


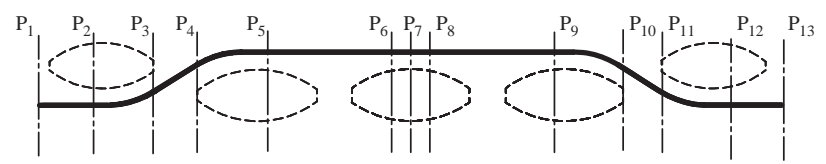

Fig. 12. Planes that define control points on the trajectory.
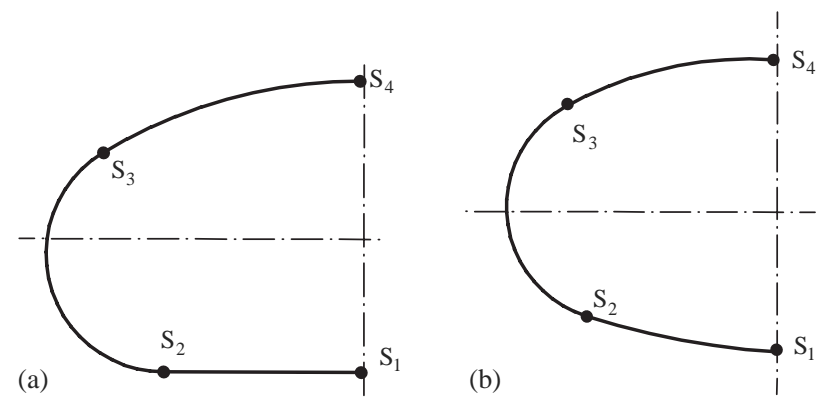

Fig. 13. Symmetrical cross section of a yarn: (a) half-section when contact zone is flat and (b) half-section without contact.

order to define the control points on the trajectory, the yarn has been cut by planes. These planes represent the beginning and end of the contact zones, and symmetry planes of the yarn. They are presented in Fig. 12. Three types of cross section appear, depending on whether the section is symmetrical according to the vertical plane or not, with or without contact. But it can be seen in Figs. 6-8, that even though the section shape changes along the yarn, variations of yarn thickness and width are small enough to be neglected. The yarn shape changes but remains in the same envelope rectangle. Moreover, refusing to consider this assumption would lead to a much more complicated model that would be very difficult to identify.

Between planes P2-P3, P4-P5, P9-P10, P11-P12, there is a contact between two yarns (Fig. 12). The transverse yarn altitude changes (top to bottom, bottom to top). The cross section is necessarily dissymmetric according to the vertical plane (Fig. 9). This section may be represented by four conics, with associated simplified forms that will be easier to identify for specific fabrics (Fig. 9a):

- Between planes P6-P8, there is a contact between two yarns but the transverse one is straight before and after the contact (Fig. 12). The cross section is symmetric according to the vertical plane. The contact zone is flat. The half section may be modeled by two conics and a segment (Fig. 13a). The simplified forms (pointed and flat lateral zones) can be associated as well.

- Between planes P1-P2, P3-P4, P5-P6, P8-P9, P10-P11, P12-P13, no contact occurs, yarn section is symmetric (Fig. 12). The half-section can be modeled by three conics (Fig. 13b).

The 3D model of the yarn is reconstructed by CAD software PROEngineer ${ }^{\circledR}$. The elementary cell of fabric is obtained by assembling $m+n$ yarns. The result is presented in Fig. 14a. Model consistency can be seen on the different cross sections presented in Fig. 14b. We have defined a geometrical consistent $3 \mathrm{D}$ model that is able to represent any $2 \mathrm{D}$ fabric, or at least those under consideration. The following developments consist in the parameterization of the model and its identification from a real fabric. All illustrations are based on a twill $3 * 2$. 

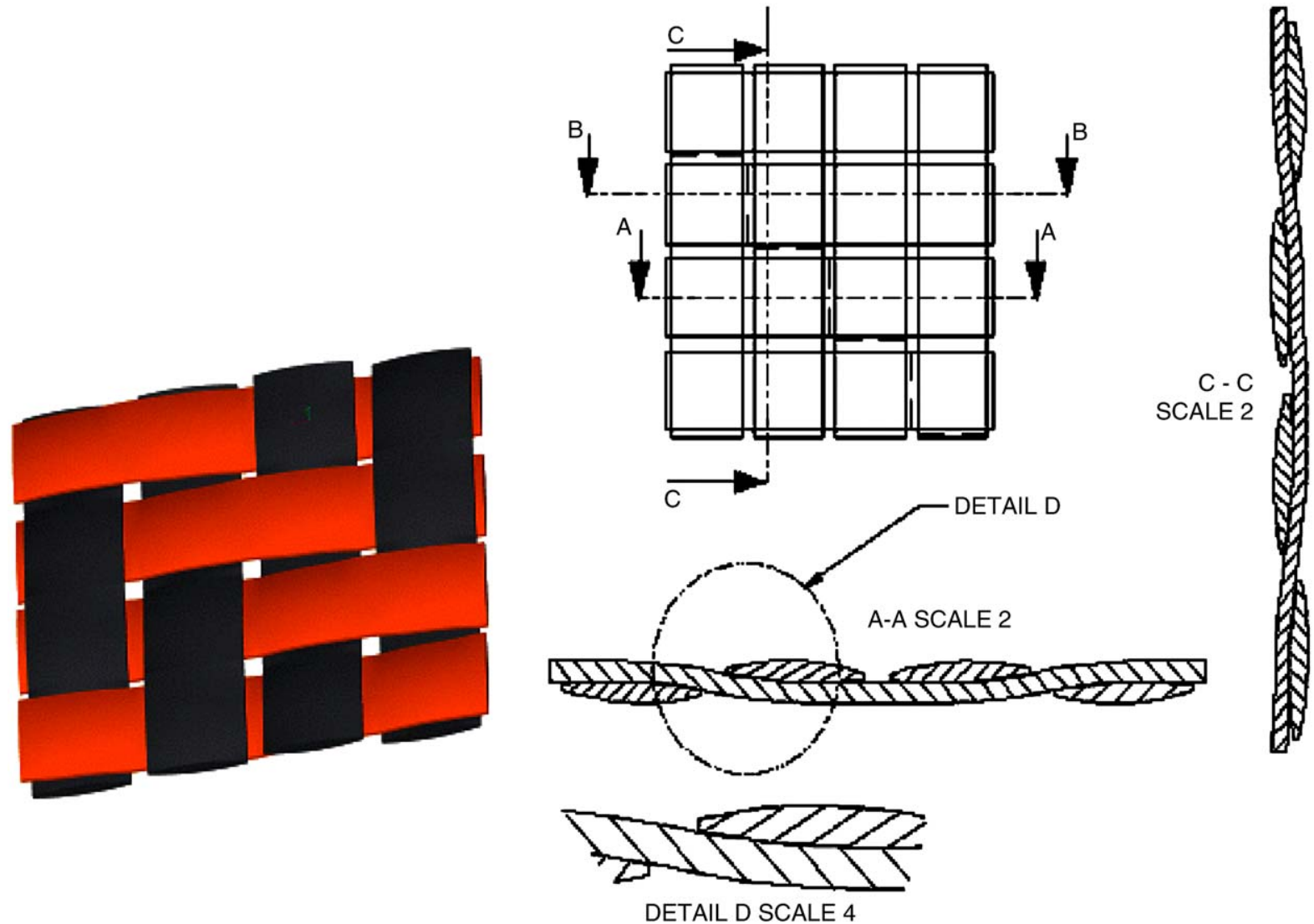

B - B SCALE 2

(a)

(b)

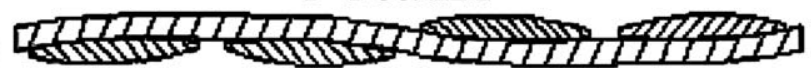

Fig. 14. 3D model of a carbon twill $2 * 2$ : (a) 3D model of a carbon twill $2 * 2$ and (b) transverse cut of a carbon twill $2 * 2$.

\section{Model identification}

\subsection{Trajectory}

The trajectory of the model previously defined is symmetrical with plane P7 (Fig. 12). Cross sections of the 3D model are anti-symmetrical with this plane. So, the complete model of the elementary cell yarn may be obtained from the knowledge of half a yarn. Therefore, we will only study one half of the elementary cell yarn. Conics representing the contact zone are parabolas, because they have a simple parametric expression and an explicit expression for their length. Parameter $\alpha$ represents the yarn direction: $\alpha=1$ for the weft direction, $\alpha=2$ for the warp direction. We will first consider the trajectory of the weft direction, for which $\alpha=1$ (Figs. 15 and 16). Control points of the trajectory are defined as $M_{\alpha i}\left(\begin{array}{l}x_{\alpha i} \\ y_{\alpha i} \\ z_{\alpha i}\end{array}\right)$ in the reference frame $R\left(M_{11}, X, Y, Z\right)$ (Fig. 15). The model's geometry implies that the control points are to be defined 


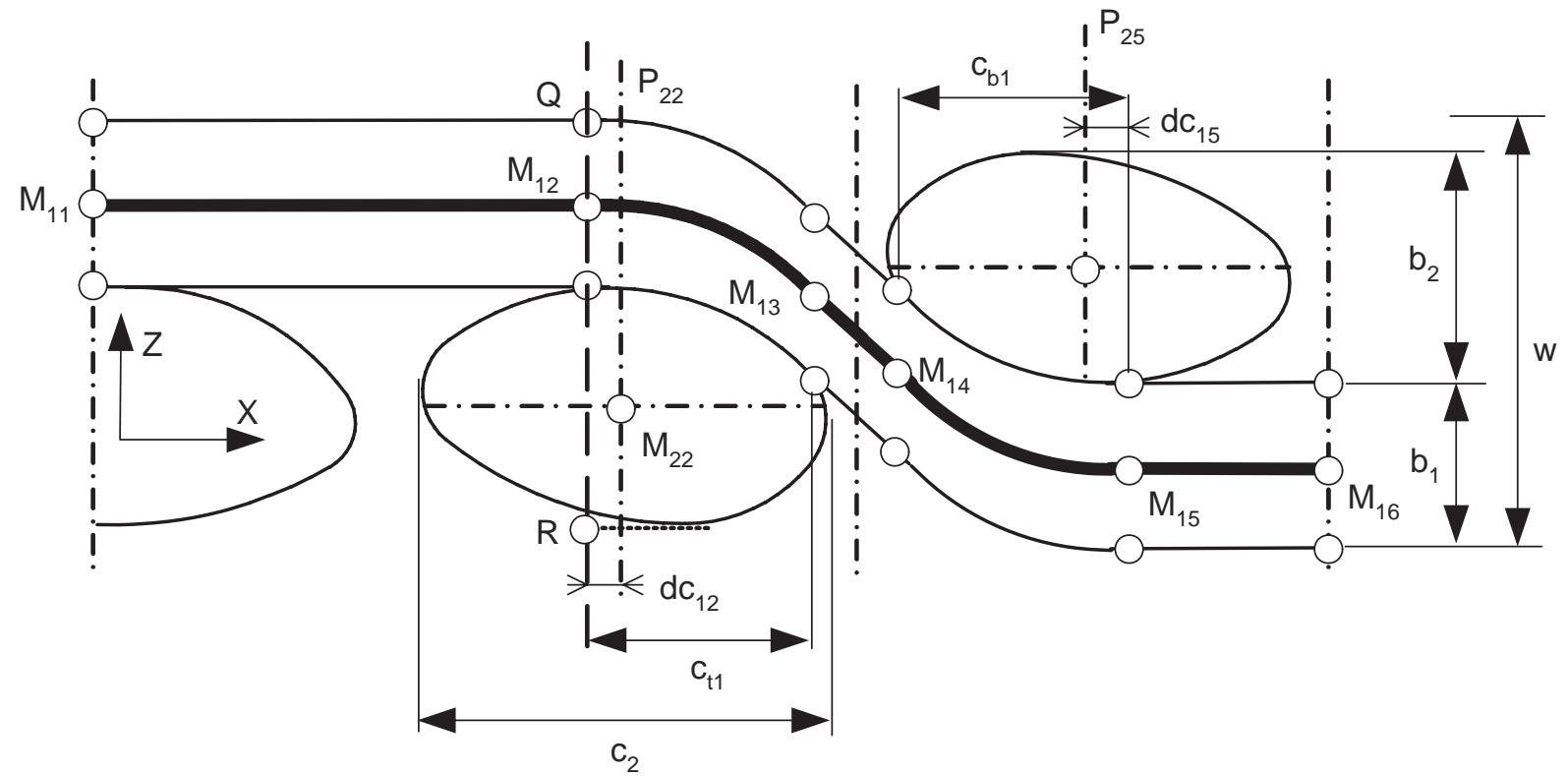

Fig. 15. Parameterization of the transverse cut (in the direction $\alpha=1$ ) of a twill $3 * 2$.

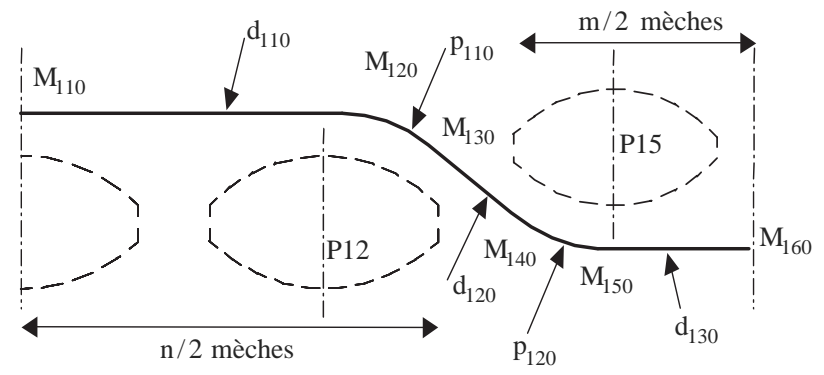

Fig. 16. Conics and control points for the trajectory of a twill $m * n$.

by eight unknown coordinates:

$$
M_{11}\left(\begin{array}{l}
0 \\
0 \\
0
\end{array}\right) M_{12}\left(\begin{array}{c}
x_{12} \\
0 \\
0
\end{array}\right) M_{13}\left(\begin{array}{c}
x_{13} \\
0 \\
z_{13}
\end{array}\right) M_{14}\left(\begin{array}{c}
x_{14} \\
0 \\
z_{14}
\end{array}\right) M_{15}\left(\begin{array}{c}
x_{15} \\
0 \\
z_{15}
\end{array}\right) M_{16}\left(\begin{array}{c}
x_{16} \\
0 \\
z_{15}
\end{array}\right) .
$$

Conics equations (two parabolas and two segments) are defined as

$$
\begin{aligned}
& p_{11} \quad z-z_{12}=p_{11}\left(x-x_{12}\right)^{2}+q_{11}, \\
& p_{12} \quad z-z_{15}=p_{12}\left(x-x_{15}\right)^{2}+q_{12}, \\
& D_{11} \quad z=0, \\
& D_{12} \quad z=d_{12} x+\delta_{12},
\end{aligned}
$$




$$
D_{13} \quad z=z_{16}
$$

Parameters of the curves can be explained as a function of the control points coordinates:

$$
\begin{aligned}
& p_{11}=\frac{z_{13}-z_{12}}{\left(x_{13}-x_{12}\right)^{2}}, \quad q_{11}=0, \\
& p_{12}=\frac{z_{14}-z_{15}}{\left(x_{15}-x_{14}\right)^{2}}, \\
& q_{12}=0, \\
& d_{12}=\frac{z_{14}-z_{13}}{x_{14}-x_{13}}, \\
& \delta_{12}=z_{14}-\frac{z_{14}-z_{13}}{x_{14}-x_{13}} x_{14} .
\end{aligned}
$$

The model is completely defined by the control point data. Two tangency conditions exist between segments and parabolas:

$$
\begin{aligned}
& 2 p_{11}\left(x_{13}-x_{12}\right)=2 \frac{z_{13}-z_{12}}{x_{13}-x_{12}}=\frac{z_{14}-z_{13}}{x_{14}-x_{13}}, \\
& 2 p_{12}\left(x_{14}-x_{15}\right)=2 \frac{z_{14}-z_{15}}{x_{14}-x_{15}}=\frac{z_{14}-z_{13}}{x_{14}-x_{13}} .
\end{aligned}
$$

Eight coordinates related by two equations define the trajectory. Six independent parameters are necessary to identify the model.

The six control points are defined as follows for the warp direction $(\alpha=2)$ :

$$
M_{21}\left(\begin{array}{c}
0 \\
0 \\
z_{21}
\end{array}\right) M_{22}\left(\begin{array}{c}
0 \\
y_{22} \\
z_{21}
\end{array}\right) M_{23}\left(\begin{array}{c}
0 \\
y_{23} \\
z_{23}
\end{array}\right) M_{24}\left(\begin{array}{c}
0 \\
y_{24} \\
z_{24}
\end{array}\right) M_{25}\left(\begin{array}{c}
0 \\
y_{25} \\
z_{25}
\end{array}\right) M_{26}\left(\begin{array}{c}
0 \\
y_{26} \\
z_{25}
\end{array}\right) .
$$

Two tangency conditions can be written and one more relation is necessary between the two trajectories to ensure the consistency of the model (Fig. 5):

$$
\begin{aligned}
& \mathrm{QR}=z_{25}-z_{21}+z_{11}-z_{15}=2\left(z_{11}-z_{21}\right), \\
& z_{21}=z_{15}-z_{25} .
\end{aligned}
$$

The warp trajectory can also be identified through six geometric parameters. The complete thread model can be identified through six parameters per direction. These parameters have to be measured on the real fabric. For most standard fabrics, the general case presented here is not necessary, and the model is much simpler. For most satins and twills, points $M \alpha 2$ and $M \alpha 5$ are very close to the vertical plane of symmetry of transverse yarns $P \alpha^{\prime} 2$ and $P \alpha^{\prime} 5\left(\alpha^{\prime}=3-\alpha\right.$; Fig. 15). A simplified model may assume that these points lay on these planes. Only four parameters per direction are necessary to identify the model in this case. Moreover, we have seen in different experimental observations, that the yarn section may be lenticular. But even when it is not (Fig. 15), the end of the contact zone (points $M \alpha 3, M \alpha 4$ ) is very close to the yarn's side (i.e. $c_{t 1}-d c_{12} \approx c_{2} / 2$ ). Parameters $c_{t \alpha}$ and $c_{b \alpha}$ can be approached as half the width of the 


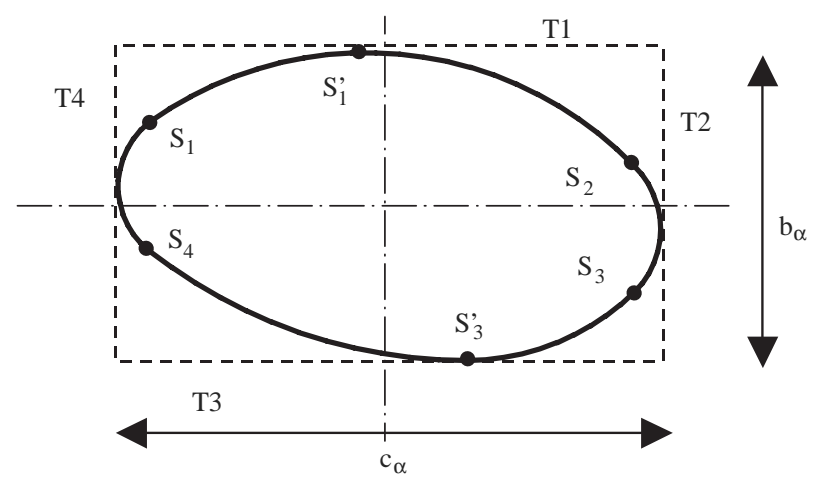

Fig. 17. Control points of a twill $m * n$ contact section.

yarn $\alpha^{\prime}$, which are quite easy to measure (Figs. 15 and 18). The combination of these two assumptions leads to a model, which can be identified with only three parameters per direction (Fig. 18; Appendix A). If the twill is balanced, only three parameters have to be identified.

As a conclusion, we have defined a simple general model of trajectory that can be identified for 2D fabrics from three (balanced fabrics) to six parameters (unbalanced twills). For specific fabrics or if many experimental parameters are available on the fabric, a more accurate model can be defined using 12 parameters. The ability of the model to change as a function of the available experimental parameters is very interesting from a practical point of view. In order to obtain the coordinates of all the control points of the trajectory, we have to solve the equations previously defined, linking these coordinates to the measured parameters (from 3 to 12, depending on the chosen model). In all cases, solving the equations posed for this model leads to the resolution of two independent, non-linear equations for unbalanced fabrics and of only one non-linear equation for balanced fabrics. The strategy of resolution is detailed in Appendix A.

\subsection{Section}

In order to complete this 3D model of yarns, it is necessary to identify cross sections at the control points. We are here presenting the identification of the most complex section (the contact section that has no symmetry). The identification of less complex sections (symmetrical, simplified models) can be deduced from this one. Four conics have to be determined: one for the contact zone between yarns, one for the contact-free zone (opposite side to the contact zone) and two lateral radiuses, the goal of which is to link the previous two conics to one another (Fig. 17). The contact conic has to be identical to the trajectory parabola to ensure the model consistency. Another parabola represents the opposite contactfree side of the yarn. Circular filets between the two parabolas model lateral parts (Fig. 17). Yarn section can be included in a rectangle envelope (Fig. 17). Two parameters have to be measured: yarn width and yarn thickness to define the yarn rectangle envelope (Fig. 17). Arc of parabola $S 1 S 2$ is defined by the consistency with the transverse trajectory. The first arc of a circle is then defined by point $S 2$, and two tangency conditions, first, with the arc of parabola in $S 2$, secondly, with the rectangle side. The second parabola may be defined by one more condition, that can either be the position of point $S^{\prime} 3$ on the rectangle's bottom side, or the condition for $S 3$ to be on the same vertical plane? as $S 2$. The last arc of a circle is defined by three tangency conditions: with the lateral side of the rectangle envelope and the 


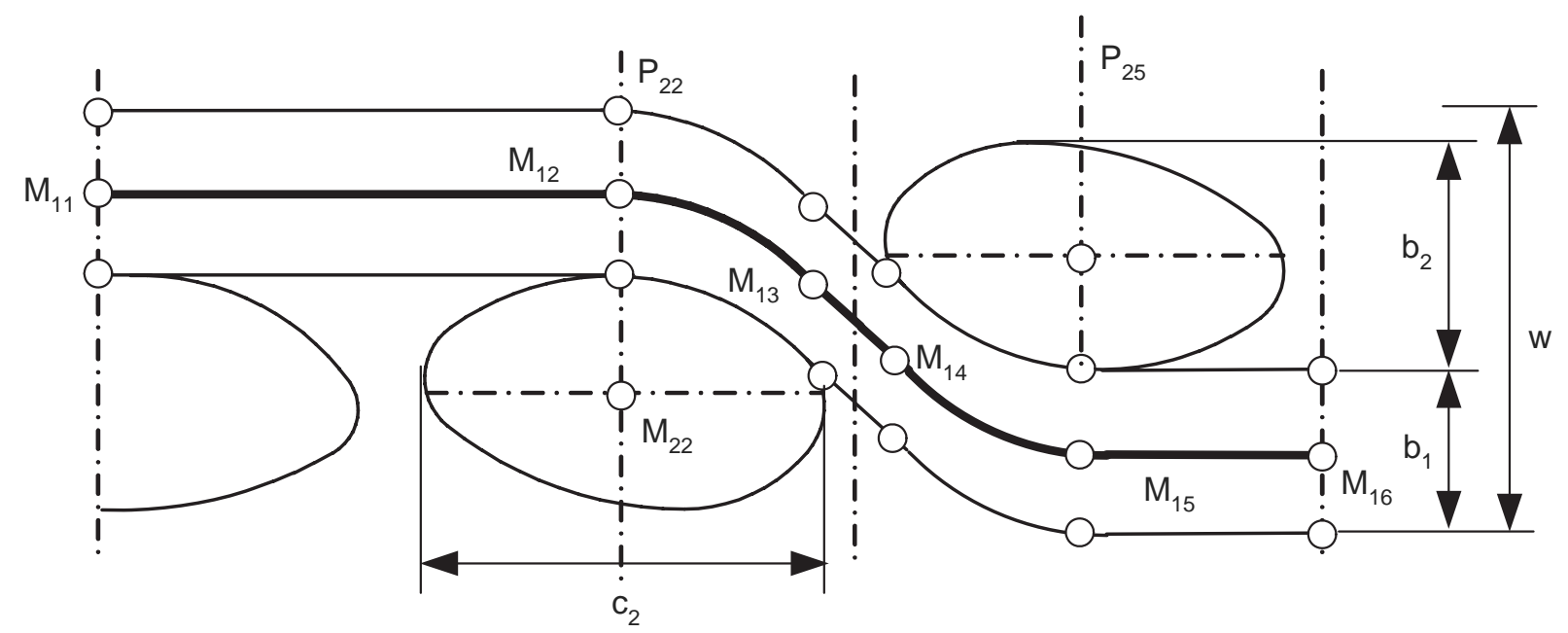

Fig. 18. Transverse cut in the direction 1 of the simplified model for a twill $m * n$.

two parabolas. Therefore, in order to define yarn cross section, three parameters have to be measured on the fabric for sections with a straight or circular lateral part. If the section shape is lenticular no lateral parts have to be considered and only two parameters (rectangle envelope) have to be identified. Width and thickness are not too difficult to get. The possible third parameter needs a more precise observation of the fabric. Here again it is interesting to notice that the model may change as a function of available experimental data. The simplest model may be identified using two parameters that are easy to measure: yarn width and thickness. Yarn widths are already involved in the determination of the trajectory in the standard model, and a relation exists between the thickness of yarns $\left(b_{1}\right.$ and $\left.b_{2}\right)$ and fabric $(w)$, and the trajectory parameters.

$$
w=\operatorname{Sup}\left\{b_{1}+\left|z_{12}-z_{15}\right|, b_{2}+\left|z_{25}-z_{22}\right|\right\} .
$$

So, only one thickness is necessary to identify the section model. Defining the yarn section only requires one additional parameter in this case. We get a very simple and realistic 3D model for fabrics, with only seven parameters for unbalanced fabrics. If a balanced fabric is considered, the relation $b_{1}=b_{2}$ can be added; no more parameters are necessary to determine the section model. Only three parameters will be necessary to identify the complete 3D model. A more sophisticated model can be used for specific fabrics for which many experimental parameters have been investigated.

The conclusion of this parameterization study is that the model is simple and consequently interesting. The standard complete 3D model of a 2D fabric may be identified by measuring from three parameters for balanced fabrics to seven parameters for unbalanced fabrics. These seven parameters may be yarn width, yarn density, crimp of each direction and thickness of the fabric. These measures are quite easy to get on fabrics, so the model will be easy to identify. For specific fabrics and if more experimental parameters are available, more sophisticated models may be identified, using from four parameters per direction (i.e. eight parameters) to nine per direction (i.e. 18 parameters). This possibility increases the model power, because it can be adapted to the user's knowledge of the fabric, and to the degree of precision he needs (Fig. 18). 


\section{3D geometrical meshing preprocessor}

The deformation of the woven cell submitted to loadings can be studied in different ways. We have already mentioned why the experimental method should be complemented with a numerical one. We are reminding here that it is difficult and time consuming to obtain enough equally distributed points in order to perform a good interpolation of tension surfaces. Especially, points on the surface boundaries, i.e. points with a very low or very high $\varepsilon_{11} / \varepsilon_{22}$ ratio, are very difficult to obtain. An analytical approach can be used [28,33], but another way consists in using finite element simulation. Durville [27] proposed to model yarns as beams, and obtained some interesting results for fabric behavior. Another approach consists in the 3D finite element simulation of the elementary cell. This technique has many advantages: local phenomena can be investigated, variations in yarn geometry can be precisely investigated, the contact zone can be observed, all types of loadings can be imposed on the elementary cell, and this technique can be applied to all fabrics that can be modeled. These are the reasons why 3D finite element simulations of the elementary mesh have been developed in our lab. The finite element analysis of fabrics is not classical because the fibrous nature of yarns composed of thousands of fibers has to be taken into account. Briefly, the finite strain mechanical behavior has to strictly follow the material direction of the fibers. This is not ensured by hypoelastic models present in most finite elements codes (based on Jaumann or Green Naghdi derivative). The particular behavior of yarns has to be implemented. This point is out off the scope of this study, but more detailed information concerning 3D simulations on elementary patterns may be found in $[2,34]$. 3D finite element simulation requires a meshed fabric. First simulations have been performed using hand made meshing [35,36], but this process is difficult and time consuming. This limits the benefits of this model, especially for sensibility studies in which the fabric geometry shall change substantially. In order to increase the performance of this model, we are proposing to use the $3 \mathrm{D}$ consistent model presented here, so as to build an automatic meshing preprocessor for $2 \mathrm{D}$ fabrics. From the parameters measured on the fabric, the preprocessor will propose a hexahedral finite element mesh of the elementary cell. Below is a description of this preprocessor and its features (Fig. 19).

First, the equations of the model are implemented in a program coded in Fortran, that contains a nonlinear equation solver (Newton with linesearch algorithm). This program reads a data file where the user enters parameters he measured on the fabric and the type of model he wants. Using the implemented equations, the parameters of the fabric 3D consistent model presented in this paper are calculated and stored in an Excel file (one file per weave type: twill, satin, plain weave). Each weave (plain, satin, twill) has been modeled with CAD software PROEngineer ${ }^{\circledR}$. These CAD models are completely parameterized and are the masters (generic models) of a family. So a new fabric model is obtained by changing the values of the calculated 3D parameters without any intervention on the CAD software. The parameters are automatically changed using PROEngineer ${ }^{\circledR}$ and Excel properties. 3D parameter values are affected through an Excel file, using the family table functions. PROEngineer ${ }^{\circledR}$ is able to read the Excel file filled by the Fortran code and generate the 3D model of the associated fabric. At this point, we have already defined an automatic geometrical preprocessor that is able to process a consistent 3D model of any 2D fabric (Fig. 19).

Validations of the process have been performed on five pieces of real fabrics: 2 balanced plain weave, 1 unbalanced glass plain weave and 2 balanced carbon twill $2 * 2$. Two types of parameters are measured on fabrics: parameters that are necessary to identify the 3D model, and other parameters, such as fabric thickness, or yarn geometry, with the aim of comparing results given by the model against those obtained through experiments. The results obtained with the model are consistent with the real values, for 


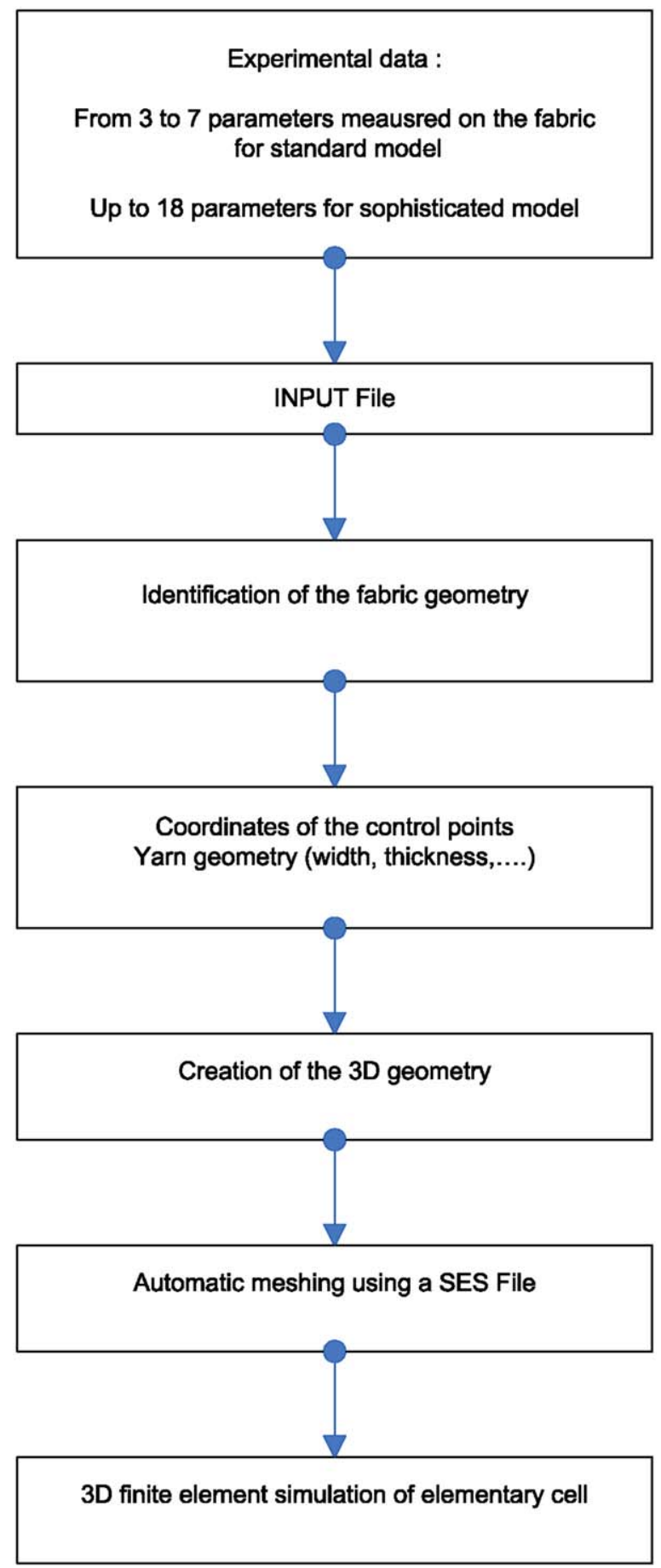

Fig. 19. Preprocessor flow chart: task organization. 

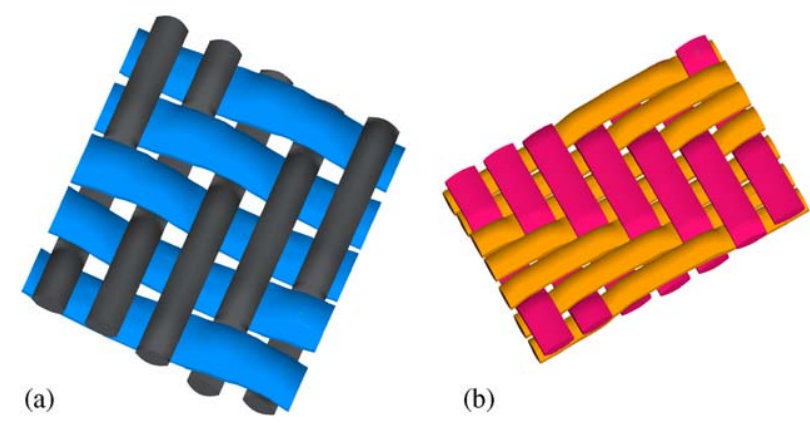

(b)

Fig. 20. 3D model of twills realized with PROEngineer: (a) 3D geometrical model of a twill $3 * 2$ and (b) 3D geometrical model of a twill $4 * 3$.

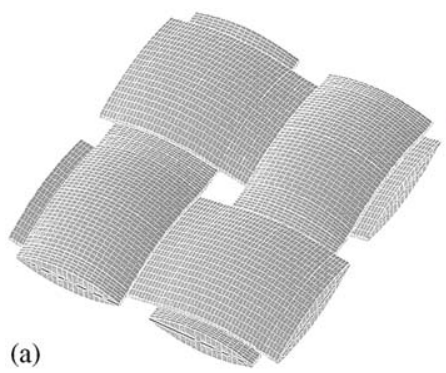

(a)

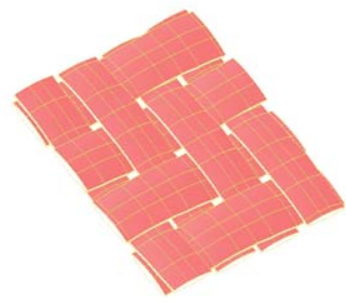

(b)

Fig. 21. 3D mesh for a plain weave fabric and a twill fabric.

instance, the ratios between measured and model thickness (for each yarn and for the fabric) for balanced, unbalanced, plain weaves and twills are less than 5\%. Many different types of fabric models have been generated, in order to confirm that any type of 2D fabrics may be processed. Two examples are presented in Fig. 20.

This geometrical preprocessor can now be used to realize meshes of elementary cells. This geometrical preprocessor can now be used to realize the mesh of elementary cells. The geometrical CAD model has to be transformed so as to obtain a hexahedral mesh of the elementary pattern. The 3D geometry of yarns is complex. In fact, we need a powerful automatic hexahedral-meshing algorithm in order to be able to mesh the fabric. We also need a program that is able to read the PROEngineer ${ }^{\circledR}$ geometry. Patran ${ }^{\circledR}$ is one of the software available in the lab that has these features. The 3D geometry is imported in Patran ${ }^{\circledR}$. The mesh of the fabric is then automated using the PCL language. Different examples of meshed fabrics are presented in Fig. 21. The mesh realized with Patran ${ }^{\circledR}$ can be imported under Abaqus [37], where simulations are carried out. The previous preprocessor coupled with $3 \mathrm{D}$ finite element analysis enables us to determine the mechanical behavior of the fabric. Mesoscopic data, such as transverse strain or the decrease of thickness and width may be analyzed with this approach. As a conclusion, a hexahedral meshing preprocessor has been developed. It will significantly increase the performance of the 3D finite element simulation. The sensitivity analysis, for instance, is completed substantially faster, and allows important investigations on the influence of the constitutive parameters of fabrics. 


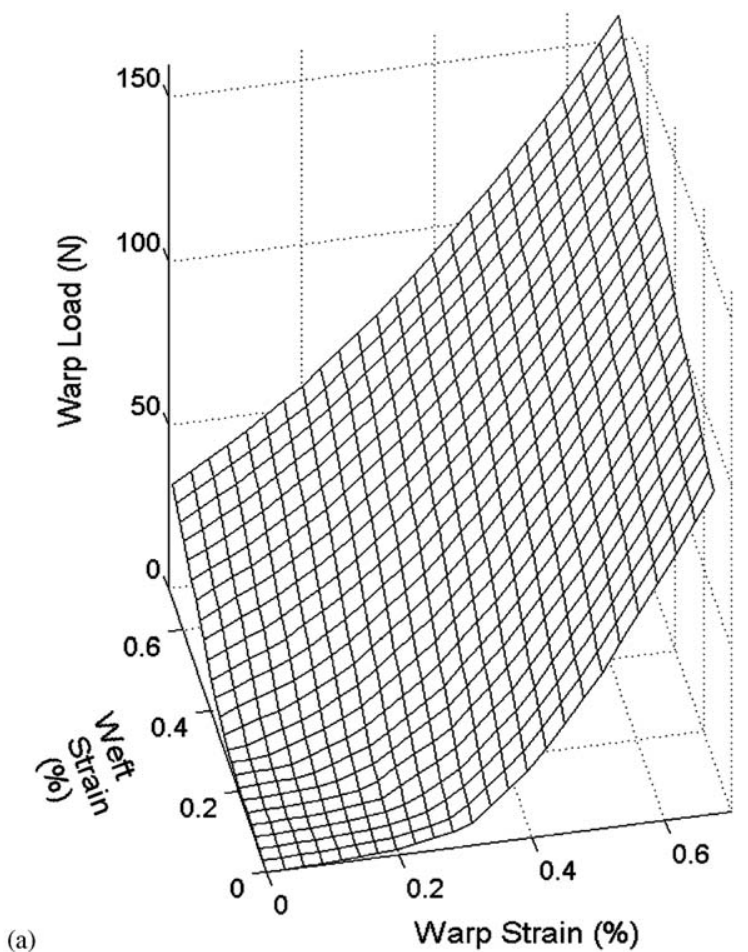

(b)

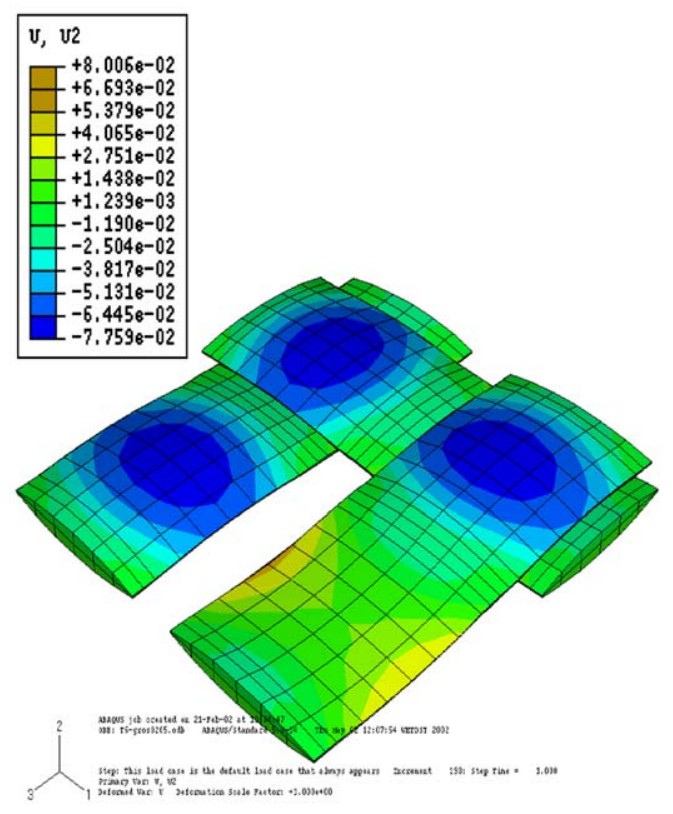

(d)

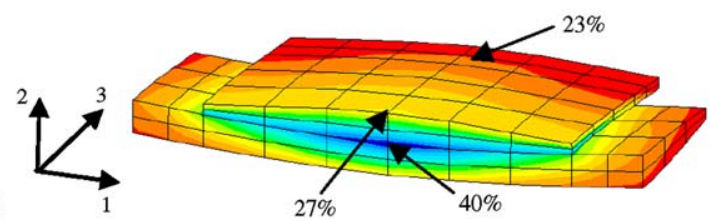

Fig. 22. Example of results obtained with the 3D finite element model of an elementary cell for glass plain weave: (a) tensile behavior surface; (b) displacements normal to the fabric plane with a yarn hidden; (c) global crushing yarns between the initial state (white) and tensed state (gray); and (d) local crushing: transverse deformation (direction 2).

\section{Finite element simulation of the elementary cell}

Both shear and biaxial tensions have been simulated. The obtained results give important information on the mechanical behavior of a fabric. Macroscopic results, such as tensile surfaces and shear curves can be obtained for different fabrics (Fig. 22a) [36]. Local data, such as the local crushing near the contact area, or local geometry variations can be observed at the meso-scale (Fig. 22b-d, [2]). The 3D finite element analysis of the elementary mesh enables us to investigate the influence of different fabric parameters. The 3D geometrical preprocessor is a very important feature enabling the efficient use of the power of these simulations. It helps obtain accurate models easily. 


\section{Conclusion}

We have defined a simple and consistent 3D geometrical model of 2D woven fabrics, which is suitable for most shapes and weavings. The standard model is defined from seven parameters for unbalanced twills, down to three parameters for balanced fabrics. But the complexity of the model geometry can vary depending on the parameters that can be measured on the fabric. This enabled us to create a 3D geometrical meshing preprocessor. Using PROEngineer ${ }^{\circledR}$ and Patran ${ }^{\circledR}$, we are able to obtain a hexahedral mesh of the fabric geometrical model. This is a very important tool that will help the development of 3D finite element simulations of elementary cells. Future developments may include other forms of fabrics, such as $2.5 \mathrm{D}$ or $3 \mathrm{D}$ fabrics, but this is much more complex.

\section{Acknowledgements}

We would like to express our sincere gratitude to the University of Massachusetts Lowell, and especially to Prof. Julie Chen and her students for their kind support and dedication to this project.

\section{Appendix A}

\section{A.1. Measured parameters}

\section{A.1.1. Most sophisticated model}

The six parameters per direction chosen to be measured for each direction are the following:

- yarn density $d_{\alpha}$ with: $a_{\alpha}=1 / d_{\alpha}$;

- position of the contact point/yarn side: $c_{t \alpha}, c_{b \alpha}$;

- crimp: $e_{\alpha}$;

- position of the contact point/vertical plane of the section: $d_{c \alpha 2}, d_{c \alpha 5}$.

\section{A.1.2. Standard model}

The three parameters per direction selected for measure for each direction are the following:

- yarn density $d_{\alpha}$ with: $a_{\alpha}=1 / d_{\alpha}$;

- yarn width: $c_{\alpha}$;

- crimp: $e_{\alpha}$.

Position of the contact point/yarn side: $c_{t \alpha}=c_{t \alpha}=c_{\alpha^{\prime}} / 2, \alpha^{\prime}=3-\alpha$.

Position of the contact point/vertical plane of the section: $d_{C \alpha 2}=d_{C \alpha 5}=0$.

\section{A.2. Equations}

The model's geometry results in the following equations:

$$
x_{16}=\frac{m+n}{2} a_{2}
$$




$$
\begin{aligned}
& y_{26}=\frac{m+n}{2} a_{1}, \\
& x_{15}=\frac{n+1}{2} a_{2}-d c_{25}, \\
& y_{25}=\frac{n+1}{2} a_{1}-d c_{15}, \\
& x_{13}=\frac{n-1}{2} a_{2}+\frac{c_{b 2}}{2}, \\
& y_{23}=\frac{n-1}{2} a_{1}+c_{t 1}, \\
& x_{14}=\frac{n+1}{2} a_{2}-\frac{c_{t 2}}{2}, \\
& x_{24}=\frac{n+1}{2} a_{1}-\frac{c_{b 1}}{2}, \\
& x_{12}=\frac{n-1}{2} a_{2}+d c_{22}, \\
& y_{21}=\frac{n-1}{2} a_{1}+d c_{12}, \\
& L_{1}=\frac{n+m}{2}\left(1+e_{1}\right) a_{2}, \\
& L_{2}=\frac{n+m}{2}\left(1+e_{2}\right) a_{1} .
\end{aligned}
$$

These equations give the expression of the coordinates and the elementary thread length as a function of the measured data.

\section{A.2.1. Weft direction}

Length of the elementary thread is given by

$$
L_{1}=L_{d 11}+L_{d 12}+L_{d 13}+L_{p 11}+L_{p 12}
$$

with

$$
\begin{aligned}
& L_{p 11}=\frac{1}{4 p_{11}} \operatorname{Arcsinh}\left(2 p_{11}\left(x_{13}-x_{12}\right)\right)+\frac{\left(x_{13}-x_{12}\right)}{2} \sqrt{1+4 p_{11}^{2}\left(x_{13}-x_{12}\right)^{2}} \\
& L_{p 12}=\frac{1}{4 p_{11}} \operatorname{Arcsinh}\left(2 p_{12}\left(x_{15}-x_{14}\right)\right)+\frac{x_{15}-x_{14}}{2} \sqrt{1+4 p_{12}^{2}\left(x_{15}-x_{14}\right)^{2}} \\
& L_{d 11}=x_{12} \\
& L_{d 12}=\sqrt{\left(x_{14}-x_{13}\right)^{2}\left(1+d_{12}\right)}=\sqrt{\left(x_{14}-x_{13}\right)^{2}\left(1+4 p_{11}^{2}\left(x_{13}-x_{12}\right)^{2}\right)} \\
& L_{d 13}=x_{16}-x_{15} .
\end{aligned}
$$




\section{A.2.2. Warp direction}

$$
\begin{aligned}
& L_{2}=L_{d 21}+L_{d 22}+L_{d 23}+L_{p 21}+L_{p 22}, \\
& L_{p 21}=\frac{1}{4 p_{21}} \operatorname{Arcsinh}\left(2 p_{21}\left(y_{23}-y_{22}\right)\right)+\frac{\left(y_{23}-y_{22}\right)}{2} \sqrt{1+4 p_{21}^{2}\left(y_{23}-y_{22}\right)^{2}}, \\
& L_{p 22}=\frac{1}{4 p_{21}} \operatorname{Arcsinh}\left(2 p_{21}\left(y_{25}-y_{24}\right)\right)+\frac{\left(y_{25}-y_{24}\right)}{2} \sqrt{1+4 p_{21}^{2}\left(y_{25}-y_{24}\right)^{2}}, \\
& L_{d 21}=y_{22}, \\
& L_{d 22}=\sqrt{\left(y_{24}-y_{23}\right)^{2}\left(1+d_{22}\right)}=\sqrt{\left(y_{24}-y_{23}\right)^{2}\left(1+4 p_{21}^{2}\left(y_{23}-y_{22}\right)^{2}\right),} \\
& L_{d 23}=y_{26}-y_{25} .
\end{aligned}
$$

\section{A.3. Equation system solving}

The length of thread $\alpha$ is expressed as a function of the coordinates and $p_{\alpha_{1}} \cdot p_{\alpha_{1}}$ is obtained by solving the two independent non-linear equations (A.11) and (A.12). Tangency conditions give:

$$
\begin{aligned}
& p_{12}=\frac{x_{13}-x_{12}}{x_{14}-x_{15}} p_{11}, \\
& p_{22}=\frac{y_{23}-y_{22}}{y_{24}-y_{25}} p_{21}, \\
& z_{13}=z_{12}+p_{11}\left(x_{13}-x_{12}\right)^{2}, \\
& z_{14}=z_{13}+2 p_{11}\left(x_{13}-x_{12}\right)\left(x_{14}-x_{13}\right), \\
& z_{15}=z_{14}-p_{12}\left(x_{14}-x_{15}\right)^{2} .
\end{aligned}
$$

If

$$
\begin{aligned}
& z_{2 j}^{\prime}=z_{2 j}-z_{21}, \quad j=1,5, \\
& z_{21}^{\prime}=z_{22}^{\prime}=0, \\
& z_{23}^{\prime}=p_{21}\left(y_{23}-y_{22}\right)^{2}, \\
& z_{24}^{\prime}=z_{23}^{\prime}+2 p_{21}\left(y_{23}-y_{22}\right)\left(y_{24}-y_{23}\right), \\
& z_{25}^{\prime}=z_{24}-p_{22}\left(y_{24}-y_{25}\right)^{2},
\end{aligned}
$$

(14) gives:

$$
\begin{aligned}
& z_{21}=\frac{1}{2}\left(z_{15}-z_{25}^{\prime}\right), \\
& z_{2 j}=z_{2 j}^{\prime}+z_{21} .
\end{aligned}
$$




\section{References}

[1] E.H. Glaessgen, C.M. Pastore, O.H. Griffin, A. Birger, Geometrical and finite element modelling of textile composites, in: Composites Part B, vol. 27B, 1996, pp. 43-50.

[2] A. Gasser, P. Boisse, S. Hanklar, Analysis of the mechanical behaviour of dry fabric reinforcements. 3D simulations versus biaxial tests, Comput. Mater. Sci. 17 (1) (2000) 7-20.

[3] X.Q. Peng, J. Cao, A dual homogenization and finite element approach for material characterization of textile composites, in: Composites Part B, vol. 33(1), 2002, pp. 45-56.

[4] X. Yu, L. Zhang, Y.-W. Mai, Modelling and finite element treatment of intra-ply shearing of woven fabric, J. Mater. Process. Technol. 138 (2003) 47-52.

[5] B. Zouari, F. Dumont, J.L. Daniel, P. Boisse, Analyses of woven fabric shearing by optical method and implementation in a finite element, in: Proceedings of the Sixth International ESAFORM Conference on Material Forming, Salerno, Italy, 2003, pp. 875-878.

[6] A. Cherouat, J.L. Billoët, Mechanical and numerical modelling of composite manufacturing processes deep-drawing and laying-up of thin pre-impregnated woven fabrics, J. Mater. Process. Technol. 118 (1-3, 3) (2001) 460-471.

[7] J. Chen, D.S. Lussier, A. Sherwood, J. Cao, X.Q. Peng, The relationship between materials characterization methods and material models for stamping of woven fabric/thermoplastic composites, in: Proceedings of the Fourth International ESAFORM Conference on Material Forming, Liège, Belgium, 2001, pp. 127-130.

[8] P. Boisse, M. Borr, K. Buet, A. Cherouat, Finite element simulations of textile composite forming including the biaxial fabric behaviour, Composites Part B 28B (4) (1997) 453-464.

[9] E.A.D. Lamers, S. Wijskamp, R. Akkerman, Drape modelling of multi-layered composites, in: Proceedings of the Sixth International ESAFORM Conference on Material Forming, Salerno, Italy, 2003, pp. 323-326.

[10] E.B. Belov, S.V. Lomov, I. Verpoest, T. Peters, D. Roose, K. Hoes, H. Sol, R. Parnas, Modelling of permeability of textile reinforcements: Lattice Boltzmann method, Proceedings of the ECCM-10, CD edition, Bruge, 2002.

[11] C. Binetruy, B. Hilaire, J. Pabiot, The interaction between flows occurring inside and outside fabric tows during RTM, Compos. Sci. Technol. 57 (1997) 587-596.

[12] F. Boust, R. Torres Carrot, E. Flavin, A. Poitou, Procédé RTM: modelisation et mesure de la perméabilité des renforts fibreux, Revue des composites et matériaux avancés 12 (3) (2002).

[13] R. Gauvin, F. Trochu, Y. Lemenn, L. Diallo, Permeability measurement and flow simulation through fibre reinforcement, Ploym. Compos. 17 (1996) 34-42.

[14] S. Bickerton, P. Simaeck, S.E. Guglielmi, S.G. Advani, Investigation of draping and its effects on the mold filling process during manufacturing of a compound curved composite part, Composites Part A 28A (1997) 801-816.

[15] S. Kawabata, M. Niwa, H. Kawai, The finite-deformation theory of plain-weave fabrics, Part I: The biaxial deformation theory, J. Text. Inst. 64 (2) (1973) 21-46.

[16] A. Bulusu, J. Chen, Modelling of the unit cell geometry of twill weave fabrics during shear deformation, in: Proceedings of the Fifteenth Technical Conference American Society for Composites, College Station, TA, USA, 2000, pp. 66-75.

[17] S.V. Lomov, A.V. Gusakov, G. Huysmans, A. Prodomou, I. Verpoest, Textile geometry preprocessor for meso-mechanical models of woven composites, Compos. Sci. Technol. 60 (11) (2000) 2083-2095.

[18] F. Robitaille, A.C. Long, I.A. Jones, C.D. Rudd, Automatically generated geometric descriptions of textile and composite unit cells, Composites Part A 34 (4) (2003) 303-312.

[19] F. Scardino, An introduction to textile structures and their behavior, in: T.W.C.a.F.K. Ko (Ed.), Textile Structural Composites, 1989, pp. 1-24.

[20] B. Hagege, P. Boisse, J.L. Billoet, Specific simulation tool for the shaping process of knitted reinforcements, in: Proceedings of the Sixth International ESAFORM Conference on Material Forming, Salerno, Italy, 2003, pp. 871-874.

[21] P. Boisse, K. Buet, A. Gasser, J. Launay, Meso-Macro mechanical behavior of textile reinforcements of thins composites, Compos. Sci. Technol. 61 (3) (2001) 395-401.

[22] K. Buet-Gautier, P. Boisse, Experimental analysis and models for biaxial mechanical behavior of composite woven reinforcements, Exp. Mech. 41 (3) (2001) 260-269.

[23] S. Kawabata, Nonlinear mechanics of woven and knitted materials, in: T.W. Chou, F.K. Ko (Eds.), Textile Structural Composites, Elsevier, Amsterdam, 1989, pp. 67-116.

[24] B. Ben Boubaker, B. Haussy, J.F. Ganghoffer, Discrete models of woven structures considering yarn interactions - application to shape forming analysis, in: Proceedings of the Seventh Esaform, 2004, Trondheim, Norway. 
[25] F.T. Peirce, The geometry of cloth structure, J. Text. Inst. 28 (1937) 45-96.

[26] J. Kuhn, P. Charalambides, Modeling of plain weave fabric composite geometry, J. Compos. Mater. 33 (3) (1999) $188-220$.

[27] D. Durville, Modélisation par éléments finis des propriétés mécaniques de structures textiles: de la fibre au tissu, Revue Européenne des Eléments Finis 11 (2-3-4) (2002) 463-477 (in French).

[28] S.V. Lomov, A.V. Gusakov, G. Huysmans, A. Prodomou, I. Verpoest, Textile geometry preprocessor for meso-mechanical models of woven composites, Compos. Sci. Technol. 60 (11) (2000) 2083-2095.

[29] S.V. Lomov, T. Truong Chi, I. Verpoest, T. Peeters, D. Roose, P. Boisse, A. Gasser, Mathematical modelling of internal geometry and deformability of woven preforms, Int. J. Form. Processes 6 (3-4) (2003).

[30] S.M. Bishop, Strength and failure of woven carbon-fiber reinforced plastics for high performance applications, in: T.W.C.a.F.K. Ko (Ed.), Textile Structural Composites, 1989, pp. 173-207.

[31] K. Searles, G. Odegard, M. Kumosa, Micro- and mesomechanics of 8-harness satin woven fabric composites: I-evaluation of elastic behavior, Composites Part A: Appl. Sci. Manuf. 32 (11) (2001) 1627-1655.

[32] F. Dumont, Contribution á l'expérimentation et á la modélisation du comportement mécanique de renforts de composites tissés, Ph.D. Thesis, the University of Paris, 6, 2003 (in French).

[33] G. Hivet, Modelisation mésoscopique pour le comportement et la mise en forme des renforts de composites tissés, Ph.D. Thesis, University of Orléans, 2002 (in French).

[34] B. Hagege, P. Boisse, J.L. Billoet, Analysis and simulation of the constitutive behavior of fibrous reinforcements, in: Proceedings of the Seventh International ESAFORM Conference on Material Forming, Trondheim, Norway, 2004, pp. $317-320$.

[35] S. Hanklar, Modélisation mécanique et numérique du comportement des tissus de fibres, Simulation du comportement mésoscopique de la maille élémentaire, in Solides, Structures et Systémes Mécaniques. Ph.D. Thesis, University of Paris, 6, France, 1998 (in French).

[36] P. Boisse, A. Gasser, G. Hivet, Analyses of fabric behaviour: determination of the biaxial tension-strain surfaces and their use in forming simulations, Composites Part A 32 (10) (2001) 1395-1414.

[37] Hibbitt, Karlsson, Sorensen, Theoretical Manual of Abaqus Code, HKS Inc., 1997. 